\title{
Some characterizations of affinely full-dimensional factorial designs
}

\author{
Satoshi Aoki*†and Akimichi Takemura ${ }^{\ddagger \S}$ \\ December, 2008
}

\begin{abstract}
A new class of two-level non-regular fractional factorial designs is defined. We call this class an affinely full-dimensional factorial design, meaning that design points in the design of this class are not contained in any affine hyperplane in the vector space over $\mathbb{F}_{2}$. The property of the indicator function for this class is also clarified. A fractional factorial design in this class has a desirable property that parameters of the main effect model are simultaneously identifiable. We investigate the property of this class from the viewpoint of $D$-optimality. In particular, for the saturated designs, the $D$-optimal design is chosen from this class for the run sizes $r \equiv 5,6,7(\bmod 8)$.
\end{abstract}

Keywords: Affine hyperplane, $D$-optimality, fractional factorial designs, Hadamard maximal determinant problem, identifiability, indicator function, non-regular designs.

\section{Introduction}

In the literature on two-level fractional factorial designs, regular fractional factorial designs have been mainly studied both in theory and applications. The reason is that properly chosen regular fractional factorial designs have many desirable properties of being balanced and orthogonal. In addition, the regular fractional factorial designs are easily constructed and used based on important concepts such as resolution and aberration. An elegant theory based on the linear algebra over $\mathbb{F}_{2}$ is well established for regular two-level fractional factorial designs. See [26] for example.

On the other hand, non-regular designs have also been receiving attention of researchers over the years, in particular for some specific topics such as Plackett-Burman

\footnotetext{
*Department of Mathematics and Computer Science, Kagoshima University

${ }^{\dagger}$ CREST, JST

${ }^{\ddagger}$ Graduate School of Information Science and Technology, University of Tokyo

${ }^{\S}$ CREST, JST
} 
designs, Hall's designs and mixed-level orthogonal arrays. However, it is very difficult to derive theoretical results for general non-regular fractional factorial designs. Suppose we have $s$ controllable factors of two-levels and want to construct a fractional factorial design with $r$ runs, where $r$ is neither a 1/2-, 1/4-, 1/8-, $\ldots$ fraction of the $2^{s}$ full factorial design. One approach to choose an $r$-runs design is to rely on various optimal criteria such as $D-, A$ - or E-optimality. However, the problem of characterizing these optimal designs for practically many values of $(s, r)$ is combinatorially very difficult. See [28] and [7] for examples of $D$-optimal saturated designs. These works, in addition to enormous literature on Hadamard matrices such as [20], indicate the difficulty of the characterization of the $D$-optimal designs and the development of simple algorithms for obtaining them. For the other criteria such as $A$ - or $E$-optimality, see [33]. Another approach for the problem of optimal selection is various extension of the minimum aberration criterion to non-regular designs. See [13] and [39] for minimum $G_{2}$-aberration, [47] for generalized minimum aberration, [46] for minimum moment aberration.

In this paper, we give a new approach for investigating general non-regular fractional factorial designs from a theoretical viewpoint. We define a class of non-regular designs, which is derived naturally from the argument of identifiability of parameters. There are some other works considering such a classification of designs. [1] gives a classification of fractional factorial designs with simple structure in view of their indicator functions. The indicator function, first introduced in [17, has become a powerful tool for studying general non-regular fractional factorial designs. See [8] and 25] for example. Since our work is motivated by the indicator function approach, we also consider properties of the indicator function of the designs in our proposed class.

The construction of this paper is as follows. In Section 2, we define a new class of the two-level non-regular fractional factorial designs and investigate its property. In Section 3 , we consider relations between the class and $D$-optimal designs. In particular we pay special attention to the saturated designs. Finally in Section 4, we give some discussion.

\section{Definition of an affinely full-dimensional factorial design}

Suppose there are $s$ controllable factors of two levels. Let $\mathcal{D}$ be the $2^{s}$ full factorial design with levels being -1 and $1 . \mathcal{D}$ is written as

$$
\mathcal{D}=\{-1,1\}^{s}=\left\{\left(x_{1}, \ldots, x_{s}\right) \mid x_{1}^{2}=\cdots=x_{s}^{2}=1\right\} .
$$

A fractional factorial design $\mathcal{F}$ (without replication) is a subset of $\mathcal{D}$. Let $r$ be the run size of $\mathcal{F}$. Therefore $\mathcal{F}$ is a set of $r$ points in $\mathcal{D}$. Arranging the elements of $\mathcal{F}$ appropriately, we represent $\mathcal{F}$ as an $r \times(s+1)$ matrix $M \in\{-1,1\}^{r \times(s+1)}$

$$
M=\left(\begin{array}{cccc}
1 & m_{11} & \cdots & m_{1 s} \\
\vdots & \vdots & & \vdots \\
1 & m_{r 1} & \cdots & m_{r s}
\end{array}\right),
$$


where $m_{i j}$ is the level of the $j$ th factor in the $i$ th run. We call the left-most column of $M$ the " 0 -th column" and write its elements as $m_{i 0} \equiv 1, i=1, \ldots, r$. Note that $M$ is the design matrix of $\mathcal{F}$ for the main effect model. If we write $\beta_{j}$ as the parameter of the main effect of the $j$ th factor for $j=1, \ldots, s$, the linear model for the observation variable $\mathbf{Y}=\left(Y_{1}, \ldots, Y_{r}\right)^{\prime}$ is written as

$$
\begin{gathered}
\mathbf{Y}=M \beta+\varepsilon, \varepsilon \sim N_{m}\left(\mathbf{0}, \sigma^{2} I\right), \\
\beta=\left(\beta_{0}, \beta_{1}, \ldots, \beta_{s}\right)^{\prime},
\end{gathered}
$$

where $\beta_{0}$ is the parameter for intercept. We put $m_{1 j}=1$ for $j=1, \ldots, s$ without loss of generality (by relabeling the two levels of each factor). We also assume that each column $\left(m_{1 j}, \ldots, m_{r j}\right)^{\prime}, j=1, \ldots, s$, contains at least one -1 .

The indicator function $f$ of $\mathcal{F}$ is defined in [17] as a function on $\mathcal{D}$ such that

$$
f(\mathbf{x})= \begin{cases}1, & \text { if } \mathbf{x} \in \mathcal{F}, \\ 0, & \text { if } \mathbf{x} \in \mathcal{D} \backslash \mathcal{F} .\end{cases}
$$

Following [17, we define contrasts $X_{I}(\mathbf{x})=\prod_{i \in I} x_{i}$ on $\mathcal{D}$ for $I \in \mathcal{P}$, where $\mathcal{P}$ is the set of all subsets of $\{1, \ldots, s\}$. A fundamental fact in the theory of the experimental design is that $\left\{X_{I}, I \in \mathcal{P}\right\}$ forms an orthogonal basis of the set of all real-valued functions on $\mathcal{D}$. The indicator function $f$ of $\mathcal{F}$ is then written as the polynomial form

$$
f(\mathbf{x})=\sum_{I \in \mathcal{P}} b_{I} X_{I}(\mathbf{x}) .
$$

Since $x_{i}^{2}=1$ on $\mathcal{D}$ for $i=1, \ldots, s$, the above expression is square-free and is unique on $\mathcal{D}$.

A regular $2^{s-k}$ fractional factorial design $\mathcal{A} \subset \mathcal{D}$ is generated by $k$ linearly independent generating relations

$$
X_{I_{1}}(\mathbf{x})=1, \ldots, X_{I_{k}}(\mathbf{x})=1,
$$

i.e. $\mathcal{A}=\left\{\mathbf{x} \in \mathcal{D} \mid X_{I_{\ell}}(\mathbf{x})=1, \ell=1, \ldots, k\right\}$. $\mathcal{A}$ contains $2^{s-k}$ points. Note that the right hand sides of the relations (4) reflect the assumption $m_{1 j}=1$ for $j=1, \ldots, s$. In general, we can take $X_{I_{\ell}}(\mathbf{x})=-1$ instead of $X_{I_{\ell}}(\mathbf{x})=1$ in (4). This just depends on the labeling of two levels for each factor. From randomization viewpoint, given the labelings of two levels of each factor, it is desirable to choose 1 or -1 randomly for each generating relation in (4).

Now we define a class of non-regular fractional factorial designs.

Definition 2.1. A non-regular fractional factorial design $\mathcal{F}$ is called an affinely fulldimensional factorial design if there is no regular fractional factorial design $\mathcal{A}$ satisfying $\mathcal{F} \subsetneq \mathcal{A}$. Conversely, a non-regular fractional factorial design $\mathcal{F}$ is called a subset fractional factorial design if there is some regular fractional factorial design $\mathcal{A}$ satisfying $\mathcal{F} \subsetneq \mathcal{A}$.

The above definition gives a new class of the non-regular fractional factorial designs. Any fractional factorial design with $r>2^{s-1}$ is an affinely full-dimensional factorial 
design. The merit of the definition might not be clear at a glance. One of the properties of the affinely full-dimensional factorial design is the simultaneous identifiability of the parameters in the model (2). Note that the least squares estimator of $\beta$ is written as $\hat{\beta}=\left(M^{\prime} M\right)^{-1} M^{\prime} \mathbf{y}$ for the observation $\mathbf{y}$. We call $\beta$ is simultaneously identifiable if $M^{\prime} M$ is a non-singular matrix. We also note that, for regular fractional factorial designs, the singularity of $M^{\prime} M$ simply corresponds to a confounding relation of the main effects. On the other hand, for the non-regular designs, the relation is not obvious. We clarify this point in the following lemma.

Lemma 2.1. If $\mathcal{F}$ is an affinely full-dimensional factorial design, then $M^{\prime} M$ is nonsingular, i.e., $\beta$ is simultaneously identifiable.

Proof. We show the contraposition. Suppose the columns of $M$ are linearly dependent. This dependence relation is preserved by the following operation: subtract the left-most column $(1, \ldots, 1)^{\prime}$ of $M$ from the other $s$ columns and then divide the $s$ columns by -2 . By this operation, 1 is mapped to 0 and -1 is mapped to 1 . Denote the resulting matrix by $\widetilde{M}=\left\{\widetilde{m}_{i j}\right\}$. Then $\widetilde{m}_{i j}=\left(1-m_{i j}\right) / 2$ for $j \geq 1$ and $\widetilde{m}_{i 0} \equiv 1$ for $i=1, \ldots, r$. From the assumption of linearly dependence there exists some integer vector $\mathbf{c}=\left(c_{0}, c_{1}, \ldots, c_{s}\right)^{\prime}$ satisfying

$$
\widetilde{M} \mathbf{c}=\mathbf{0} .
$$

Considering the modulo 2 reduction of (15), we have

$$
\widetilde{M} \mathbf{c}=\mathbf{0}(\bmod 2),
$$

where the odd elements and the even elements of $\mathbf{c}$ are replaced by 1 and 0 , respectively. Here we can assume that there exists an odd element of $\mathbf{c}$ in (5), since if every element of $\mathbf{c}$ is even then we can divide (5) by the power of 2 in the factorization of the greatest common divisor of the elements of c. Moreover $c_{0}=0$, since the first row of $\widetilde{M}$ is $(1,0, \ldots, 0)$. Then (6) implies that there are even 1's in $\left\{\widetilde{m}_{i j} \mid c_{j}=1, j \geq 1\right\}$ for $i=1, \ldots, r$, or equivalently there are even -1 's in $\left\{m_{i j} \mid c_{j}=1, j \geq 1\right\}$ for $i=1, \ldots, r$. Therefore $X_{I}(\mathbf{x})=1$ holds for $I=\left\{j \mid c_{j}=1, j \geq 1\right\}$.

Q.E.D.

From this lemma, we also have the following corollary.

Corollary 2.1. Let $M$ be a design matrix of a non-regular fractional factorial design. If $M^{\prime} M$ is singular, then the design is a subset fractional factorial design.

The proof of Lemma 2.1 clarifies the geometrical meaning of the class. By the correspondence $(1,-1) \leftrightarrow(0,1), X_{I}\left(x_{1}, \ldots, x_{s}\right)=1,\left(x_{1}, \ldots, x_{s}\right) \in \mathcal{D}$ if and only if

$$
c_{1} \widetilde{x}_{1}+\cdots+c_{s} \widetilde{x}_{s}=0(\bmod 2), \quad \widetilde{x}_{j}=\left(1-x_{j}\right) / 2, j=1, \ldots, s,
$$

where

$$
c_{j}= \begin{cases}1, & \text { if } j \in I, \\ 0, & \text { if } j \notin I .\end{cases}
$$


However, as remarked earlier, design points can be chosen by $X_{I}\left(x_{1}, \ldots, x_{s}\right)=-1$. Therefore, in general there may be a constant term $c_{0}=0$ or 1 :

$$
c_{0}+c_{1} \widetilde{x}_{1}+\cdots+c_{s} \widetilde{x}_{s}=0(\bmod 2), \quad \widetilde{x}_{j}=\left(1-x_{j}\right) / 2, j=1, \ldots, s .
$$

In this sense, $\mathcal{F}$ is a proper subset of no regular fractional factorial design if and only if the points of $\mathcal{F}$, considered as a subset of $\mathbb{F}_{2}^{s}=\{0,1\}^{s}$, are not contained in any affine hyperplane of the form (7), i.e., we can form a basis of $\mathbb{F}_{2}^{s}=\{0,1\}^{s}$ as the differences of the vectors in $\mathcal{F}$. This is the reason we call the class affinely full-dimensional.

The question of determining whether a given design is an affinely full-dimensional is immediately read off from its indicator function.

Lemma 2.2. Let $\mathcal{F}$ be a fractional factorial design and (3) be its indicator function. Then $\mathcal{F}$ is an affinely full-dimensional factorial design if and only if $\left|b_{I}\right|<b_{\emptyset}$ for all $I \in \mathcal{P}$.

Proof. Obvious from Proposition 4.2 and Corollary 4.3 of [17]. Q.D.

\section{$3 \quad D$-optimality of the affinely full-dimensional facto- rial designs}

In Section 2, we define a new class of the non-regular fractional factorial designs, namely, affinely full-dimensional factorial designs. The affinely full-dimensional factorial designs have a desirable property that all the parameters are always simultaneously identifiable in the saturated model. Next problem of interest is whether this class includes good designs or not in view of various optimality criteria. In this paper, we consider $D$-optimality of the designs. For given $r$ and $s$, the $D$-optimal design is the matrix $M \in\{-1,1\}^{r \times(s+1)}$ which maximizes $\left|\operatorname{det}\left(M^{\prime} M\right)\right|$. The $D$-optimal designs minimize the generalized variance of $\hat{\beta}([33])$.

\subsection{D-optimal designs for the saturated cases}

First we consider the saturated cases, i.e., the cases of $r=s+1$. In this case, the maximization of $\left|\operatorname{det}\left(M^{\prime} M\right)\right|$ reduces to the maximization of $|\operatorname{det} M|, M \in\{-1,1\}^{r \times r}$. This problem is known as the Hadamard maximal determinant problem. Despite a century of works by mathematicians, this problem remains unanswered in general.

To investigate the relation between the maximal determinant problem and the affinely full-dimensional factorial designs, we show a basic theorem.

Theorem 3.1. A design with a design matrix $M$ is affinely full-dimensional factorial if and only if $\operatorname{det} M$ is not divisible by $2^{r}$.

We define $M$ and $\widetilde{M}$ as in the proof of Lemma 2.1. The columns of these matrices are numbered from 0 to $s=r-1$ and the rows are numbered from 1 to $r$. Note that the first row of $\widetilde{M}$ is $(1,0, \ldots, 0)$. This matrix has the following property. 
Lemma 3.1. $\operatorname{det} \widetilde{M}$ is an odd integer if and only if $M$ is a design matrix of an affinely full-dimensional factorial design.

Proof of Lemma 3.1. If $M$ is a design matrix of a subset fractional factorial design or a regular fractional factorial design, there exists $1 \leq j_{1}<\cdots<j_{p} \leq s, p \geq 2$ satisfying

$$
\prod_{\ell=1}^{p} m_{i j_{\ell}}=1
$$

for $i=1, \ldots, r$. From the definition of $\widetilde{M}$, this is equivalent to

$$
\sum_{\ell=1}^{p} \widetilde{m}_{i j_{\ell}}=0(\bmod 2)
$$

for $i=1, \ldots, r$. It means that $\widetilde{M}$ is a singular matrix in $\mathbb{F}_{2}$, i.e., $\operatorname{det} \widetilde{M}=0(\bmod 2)$.

Q.E.D.

Proof of Theorem 3.1. From the definition, we construct $\widetilde{M}$ from $M$ as follows:

- subtracting the left-most column of $M$ from the other columns,

$$
m_{i j} \leftarrow m_{i j}-m_{i 0}, i=1, \ldots, r, j=1, \ldots, s,
$$

- dividing the columns except for the left-most column by -2 ,

$$
m_{i j} \leftarrow m_{i j} /(-2), i=1, \ldots, r, j=1, \ldots, s .
$$

Note that, after the first operation, no column of $M$ is $(0, \ldots, 0)^{\prime}$ from our assumption. Therefore $(-2)^{r-1} \operatorname{det} \widetilde{M}=\operatorname{det} M$ holds. From Lemma 3.1, we have proved the theorem.

Q.E.D.

Theorem 3.1 shows that whether a given non-regular fractional factorial design is affinely full-dimensional or not is judged from its determinant. Using this characteristic, we investigate the $D$-optimal designs.

There are many literature reporting the solution of the Hadamard maximal determinant problem for specific $r$. The most basic result was given by Hadamard ([19]) as $\operatorname{det} M \leq r^{r / 2}$, where the bound is achieved only for Hadamard matrices. Paley has conjectured that a Hadamard matrix exists for every $r=0(\bmod 4)$. The lowest order for which a Hadamard matrix is not yet obtained is $r=668$ ([35]). For a design where the design matrix $M$ is a Hadamard matrix, it is a subset fractional factorial design since the bound reduces to $(4 k)^{r / 2}=2^{r} k^{r / 2}$. In fact, the product of the elements in each row is 1 from the property of Hadamard matrix. Therefore we consider the case that $r$ is not 
Table 1: Summary whether the maximal determinant matrices are affinely fulldimensional factorial design (Yes) or subset fractional factorial design (No) for $r=$ $4, \ldots, 99$. The values of the maximal determinant are given as divided by $2^{r-1}$. The values that achieve the bounds ([2], [14], [45] and [15]) are underlined.

\begin{tabular}{cc|ccc|ccc|ccc}
$r$ & class & $r$ & class & Det $/ 2^{r-1}$ & $r$ & class & Det $/ 2^{r-1}$ & $r$ & class & Det $/ 2^{r-1}$ \\
\hline 4 & No & 5 & Yes & $\underline{3}$ & 6 & Yes & $\underline{5}$ & 7 & Yes & $\underline{9}$ \\
8 & No & 9 & No & $7 \cdot 2^{3}$ & 10 & No & $\underline{9 \cdot 2^{4}}$ & 11 & No & $2^{6} \cdot 5$ \\
12 & No & 13 & Yes & $\underline{5 \cdot 3^{6}}$ & 14 & Yes & $\underline{13 \cdot 3^{6}}$ & 15 & Yes & $3^{6} \cdot 5 \cdot 7$ \\
16 & No & 17 & No & $2^{16} \cdot 5$ & 18 & No & $\underline{17 \cdot 2^{8} \cdot 2^{8}}$ & 19 & $?$ & \\
20 & No & 21 & Yes & $5^{9} \cdot 29$ & 22 & $?$ & & 23 & $?$ & \\
24 & No & 25 & No & $\underline{7 \cdot 6^{12}}$ & 26 & No & $\underline{25 \cdot 3^{12} \cdot 2^{12}}$ & 27 & $?$ & \\
28 & No & 29 & $?$ & & 30 & Yes & $\underline{29 \cdot 7^{14}}$ & 31 & $?$ & \\
32 & No & 33 & $?$ & & 34 & $?$ & & 35 & $?$ & \\
36 & No & 37 & $?$ & & 38 & Yes & $\underline{37 \cdot 9^{18}}$ & 39 & $?$ & \\
40 & No & 41 & No & $\underline{9 \cdot 10^{20}}$ & 42 & No & $\underline{41 \cdot 5^{20} \cdot 2^{20}}$ & 43 & $?$ & \\
44 & No & 45 & $?$ & & 46 & Yes & $\underline{45 \cdot 11^{22}}$ & 47 & $?$ & \\
48 & No & 49 & $?$ & & 50 & No & $\underline{49 \cdot 6^{24} \cdot 2^{24}}$ & 51 & $?$ & \\
52 & No & 53 & $?$ & & 54 & Yes & $\underline{53 \cdot 13^{26}}$ & 55 & $?$ & \\
56 & No & 57 & $?$ & & 58 & $?$ & & 59 & $?$ & \\
60 & No & 61 & Yes & $\underline{11 \cdot 15^{30}}$ & 62 & Yes & $\underline{61 \cdot 15^{30}}$ & 63 & $?$ & \\
64 & No & 65 & $?$ & & 66 & No & $\underline{65 \cdot 8^{32} \cdot 2^{32}}$ & 67 & $?$ & \\
68 & No & 69 & $?$ & & 70 & $?$ & & 71 & $?$ & \\
72 & No & 73 & $?$ & & 74 & No & $\underline{73 \cdot 9^{36} \cdot 2^{36}}$ & 75 & $?$ & \\
76 & No & 77 & $?$ & & 78 & $?$ & & 79 & $?$ & \\
80 & No & 81 & $?$ & & 82 & No & $\underline{81 \cdot 10^{40} \cdot 2^{40}}$ & 83 & $?$ & \\
84 & No & 85 & $?$ & & 86 & Yes & $\underline{85 \cdot 21^{42}}$ & 87 & $?$ & \\
88 & No & 89 & $?$ & & 90 & No & $\underline{89 \cdot 11^{44} \cdot 2^{44}}$ & 91 & $?$ & \\
92 & No & 93 & $?$ & & 94 & $?$ & & 95 & $?$ & \\
96 & No & 97 & $?$ & & 98 & No & $\underline{97 \cdot 12^{48} \cdot 2^{48}}$ & 99 & $?$ &
\end{tabular}


divisible by 4 . According to the literature reporting the maximal determinant matrices, we investigate whether the $D$-optimal design matrix is affinely full-dimensional factorial or not for $r<100$. The results are given in Table 1 , In Table 1 "?" means that the maximal determinant matrix is not obtained (or the maximality is not proved) at present. We also show the values of the maximal determinant divided by $2^{r-1}$ for $r=1,2,3$ (mod 4). We underline the values, when the values achieve the known bounds such as [2] for $r \equiv 1(\bmod 4)$, [14] and [45] for $r \equiv 2(\bmod 4)$ and [15] for $r \equiv 3(\bmod 4)$. We omit the reference for the Hadamard matrices. For the other maximal determinant matrices, see [27] for $r=5$; [44] for $r=6,7$; [16] for $r=9,10,11$; [36] for $r=9,10,15$; [45] for $r=10,26$; [18] for $r=11$; [34] for $r=13,25,50$; [12] for $r=13$; [14 for $r=14,18,26,30,38$; [10] for $r=14,18$; [9] for $r=15,74,82,90,98$; [1] and [29] for $r=15$; [41, 28] and [21] for $r=17$; [50] for $r=18,26,30,38$; [7] and [30] for $r=21$; 4] for $r=25,61$; [23] for $r=26,30,38,42,46,50,54,66$; [3], [43], 37] and [38] for $r=41$; [48] and [22] for $r=42,66$; [51] for $r=50,62$; [49] for $r=54$; [31] and [32] for $r=61$; 52] for $r=66$; [5] for $r=86$; 24] for $r=90$.

Table 1 shows an interesting periodicity. We present the following conjecture.

Conjecture 3.1. For $r=5,6,7(\bmod 8)$, D-optimal design is affinely full-dimensional factorial. For $r=0,1,2,3,4$ ( $\bmod 8)$, D-optimal design is subset fractional factorial.

Though the authors do not succeed in proving this conjecture, we do not find any counterexample at present. We consider special cases instead. For the case $r=1$ (mod 4), [2] gives a bound

$$
\operatorname{det} M \leq(2 r-1)^{1 / 2}(r-1)^{(r-1) / 2} .
$$

See also [14] and [45. This bound is achievable only if $2 r-1$ is a perfect square. In fact, for $r=5,13,25,41,61$ in Table 1, the maximum determinant achieves the bound, whereas the maximal determinant matrix has not been founded at present for $r=85$. For the cases that the bound is attained, it is easy to prove the conjecture.

Proposition 3.1. For $r=1(\bmod 8)$ and the bound (8) is attained, the maximal determinant matrix is chosen as a design matrix for the subset fractional factorial design. On the other hand, for $r=5(\bmod 8)$ and the bound (8) is attained, the maximal determinant matrix is chosen as a design matrix for the affinely full-dimensional factorial design.

Proof. Write $2 r-1=m^{2}$ and $r=4 k+1$. Then it holds

$$
(2 r-1)^{1 / 2}(r-1)^{(r-1) / 2}=m 2^{r-1} k^{2 k} .
$$

Since $m$ is an odd integer, the maximal determinant is divisible by $2^{r}$ if and only if $k$ is an even integer, i.e., $r=1(\bmod 8)$.

Q.E.D.

Similarly, for the case that $r=2(\bmod 4),[14$ and [45] give a bound

$$
\operatorname{det} M \leq 2(r-1)(r-2)^{(r-2) / 2} \text {. }
$$


In Table 1, all the maximum determinants achieve the bound for $r=6,10, \ldots, 98$ except for unsolved cases, $r=22,34,58,70,78,94$. For the cases that the bound is achieved, it is also easy to prove the conjecture.

Proposition 3.2. For $r=2(\bmod 8)$ and the bound (9) is attained, the maximal determinant matrix is chosen as a design matrix for the subset fractional factorial design. On the other hand, for $r=6(\bmod 8)$ and the bound (9) is attained, the maximal determinant matrix is chosen as a design matrix for the affinely full-dimensional factorial design.

Proof. For $r=8 k+2, k \geq 1$, the bound,

$$
2(r-1)(r-2)^{(r-2) / 2}=(r-1) k^{4 k} 2^{r+4 k-1},
$$

is divisible by $2^{r}$ since $k \geq 1$. For $r=8 k+6, k \geq 0$, the bound,

$$
2(r-1)(r-2)^{(r-2) / 2}=(r-1)(2 k+1)^{4 k+2} 2^{r-1},
$$

is not divisible by $2^{r}$ since both $r-1$ and $2 k+1$ are odd integers.

Q.E.D.

For the cases that $r=3(\bmod 4)$, though a similar bound is given by [15], the maximal determinant matrix attaining the bound is not found at present except for $r=3$.

\subsection{D-optimal designs for 4 and 5 factors cases}

Next we consider the non-saturated cases of $s=4,5$. We enumerate all the design matrices of the runs $r \leq 10$ and obtain the $D$-optimal designs. We investigate whether the $D$ optimal designs are the affinely full-dimensional factorial design or the subset fractional factorial design. The result is summarized in Table 2 and 3 . Note that the factorial design is always affinely full-dimensional for $r>8, s=4$ since $r>2^{s-1}$.

We have not yet derived any clear relation between the $D$-optimality and the affine full-dimensionality like Conjecture 3.1 at present from the results in Table 2 and 3 .

\section{Discussion}

We classify non-regular designs by whether it is a proper subset of some regular fractional factorial design (namely, subset fractional factorial design) or not (namely, affinely fulldimensional factorial design). We also give a geometrical interpretation of each class, i.e., the affinely full-dimensional factorial design is characterized as the design with the points which are not contained in any affine hyperplane. One justification of our definition is the simultaneous identifiability of the parameters for the main effect model, which is one of the most important concepts in the theory of designed experiments. Therefore, if the main purpose of the data analysis is the estimation of the parameters for the main effect model, the strategy of considering the affinely full-dimensional factorial designs is useful. 
Table 2: $D$-optimal designs for 4 factors

\begin{tabular}{|c|c|c|c|c|c|c|c|c|c|c|c|c|}
\hline \multirow[t]{2}{*}{$r$} & \multicolumn{4}{|c|}{5} & \multicolumn{4}{|c|}{6} & \multicolumn{4}{|c|}{7} \\
\hline & $x_{1}$ & $x_{2}$ & $x_{3}$ & $x_{4}$ & $x_{1}$ & $x_{2}$ & $x_{3}$ & $x_{4}$ & $x_{1}$ & $x_{2}$ & $x_{3}$ & $x_{4}$ \\
\hline \multirow{7}{*}{$\begin{array}{l}D \text {-optimal } \\
\text { design }\end{array}$} & 1 & 1 & 1 & 1 & 1 & 1 & 1 & 1 & 1 & 1 & 1 & 1 \\
\hline & 1 & 1 & -1 & -1 & 1 & 1 & 1 & -1 & 1 & 1 & 1 & -1 \\
\hline & 1 & -1 & 1 & -1 & 1 & 1 & -1 & 1 & 1 & -1 & -1 & 1 \\
\hline & -1 & 1 & 1 & -1 & 1 & -1 & -1 & -1 & 1 & -1 & -1 & -1 \\
\hline & -1 & -1 & -1 & 1 & -1 & 1 & -1 & -1 & -1 & 1 & -1 & 1 \\
\hline & & & & & -1 & -1 & 1 & 1 & -1 & 1 & -1 & -1 \\
\hline & \multirow{4}{*}{\multicolumn{4}{|c|}{$\begin{array}{c}\text { affinely full-dim. } \\
\text { none } \\
2^{8} \cdot 3^{2}\end{array}$}} & & & & & -1 & -1 & 1 & 1 \\
\hline class & & & & & \multirow{3}{*}{\multicolumn{4}{|c|}{$\begin{array}{c}\text { affinely full-dim. } \\
\text { none } \\
2^{10} \cdot 5\end{array}$}} & \multirow{3}{*}{\multicolumn{4}{|c|}{$\begin{array}{c}\text { subset FF } \\
x_{1} x_{2} x_{3}=1 \\
2^{12} \cdot 3\end{array}$}} \\
\hline relation & & & & & & & & & & & & \\
\hline $\max \left|\operatorname{det} M^{\prime} M\right|$ & & & & & & & & & & & & \\
\hline \multirow[t]{5}{*}{$r$} & \multicolumn{4}{|c|}{8} & \multicolumn{4}{|c|}{9} & \multicolumn{4}{|c|}{10} \\
\hline & $x_{1}$ & $x_{2}$ & $x_{3}$ & $x_{4}$ & $x_{1}$ & $x_{2}$ & $x_{3}$ & $x_{4}$ & $x_{1}$ & $x_{2}$ & $x_{3}$ & $x_{4}$ \\
\hline & 1 & 1 & 1 & 1 & 1 & 1 & 1 & 1 & 1 & 1 & 1 & 1 \\
\hline & 1 & 1 & 1 & -1 & 1 & 1 & 1 & -1 & 1 & 1 & 1 & -1 \\
\hline & 1 & -1 & -1 & 1 & 1 & 1 & -1 & 1 & 1 & 1 & -1 & 1 \\
\hline \multirow{6}{*}{$\begin{array}{l}D \text {-optimal } \\
\text { design }\end{array}$} & 1 & -1 & -1 & -1 & 1 & -1 & 1 & 1 & 1 & -1 & 1 & 1 \\
\hline & -1 & 1 & -1 & 1 & 1 & -1 & -1 & -1 & 1 & -1 & -1 & -1 \\
\hline & -1 & 1 & -1 & -1 & -1 & 1 & 1 & 1 & -1 & 1 & 1 & 1 \\
\hline & -1 & -1 & 1 & 1 & -1 & 1 & -1 & -1 & -1 & 1 & 1 & -1 \\
\hline & -1 & -1 & 1 & -1 & -1 & -1 & 1 & -1 & -1 & 1 & -1 & -1 \\
\hline & & & & & -1 & -1 & -1 & 1 & $\begin{array}{l}-1 \\
-1\end{array}$ & $\begin{array}{l}-1 \\
-1\end{array}$ & $\begin{array}{r}1 \\
-1\end{array}$ & $\begin{array}{r}-1 \\
1\end{array}$ \\
\hline class & \multicolumn{4}{|c|}{ regular FF } & \multicolumn{4}{|c|}{ affinely full-dim. } & \multirow{3}{*}{\multicolumn{4}{|c|}{$\begin{array}{c}\text { affinely full-dim. } \\
\text { none } \\
2^{12} \cdot 3 \cdot 7\end{array}$}} \\
\hline relation & \multicolumn{4}{|c|}{$x_{1} x_{2} x_{3}=1$} & \multicolumn{4}{|c|}{ none } & & & & \\
\hline $\max \left|\operatorname{det} M^{\prime} M\right|$ & & & & & & & & & & & & \\
\hline
\end{tabular}


Table 3: $D$-optimal designs for 5 factors

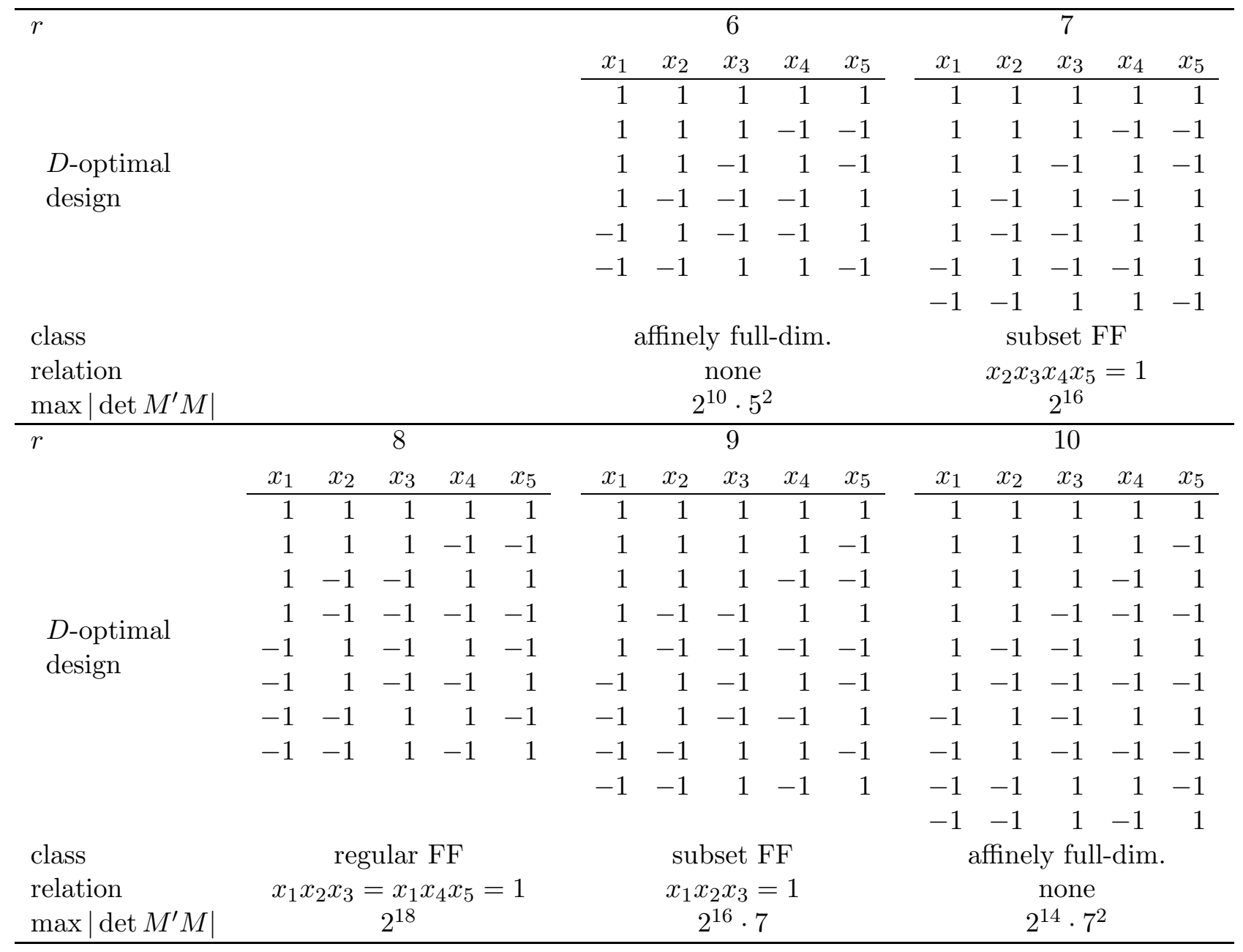


It is an interesting topic to investigate whether various "good" designs are the affinely full-dimensional factorial designs or not. In this paper, we consider this problem from the concept of $D$-optimality. In particular, from the investigation of the $D$-optimal designs for the saturated cases, interesting mod 8 periodicity is suggested. Though the conjecture is not proved at present, it holds for all the proved $D$-optimal designs under 100 runs. We believe that the conjecture interests researchers studying Hadamard maximum determinant problem.

It is also important to consider other optimal criteria such as $A$ - or $E$-optimality. As an initial investigation, we have confirmed that the $D$-optimal designs of saturated designs for $s=4,5$ in Table 2 and 3 are also $A$-optimal and $E$-optimal designs.

\section{References}

[1] N. Balakrishnan and P. Yang (2006). Classification of three-word indicator functions of two-level factorial designs. Annals of the Institute of Statistical Mathematics, 58, 595-608.

[2] G. Barba (1933). Intorno al teorema di Hadamard sui determinanti a valore massimo, Giorn. Mat. Battaglini, 71, 70-86.

[3] W. G. Bridges, M. Hall, Jr. and J. L. Hayden (1981). Codes and designs, J. Combin. Theory Ser. A, 31, 155-174.

[4] A. E. Brouwer (1983). An infinite series of symmetric designs, Math. Centrum Amsterdam Report ZW 202/83.

[5] T. Chadjipantelis and S. Kounias (1985). Supplementary difference sets and Doptimal designs for $n=2 \bmod 4$, Discrete Math., 57, 211-216.

[6] T. Chadjipantelis, S. Kounias and C. Moyssiadis (1985). Construction of D-optimal designs for $n \equiv 2 \bmod 4$ using block-circulant matrices. J. Combin. Theory Ser. A, 40, 125-135.

[7] T. Chadjipantelis, S. Kounias and C. Moyssiadis (1987). The maximum determinant of $21 \times 21(+1,-1)$-matrices and D-optimal designs. Journal of Statistical Planning and Inference, 16, 167-178.

[8] S. W. Cheng, W. Li and K. Q. Ye (2004). Blocked nonregular two-level factorial designs. Technometrics, 45, 347-351.

[9] J. H. E. Cohn (1989). On determinants with elements \pm 1 , II, Bull. London Math. Soc., 21, 36-42.

[10] J. H. E. Cohn (1994). On the number of D-optimal designs. J. Combin. Theory Ser. A, 66, 214-225. 
[11] J. H. E. Cohn (2000). Almost D-optimal designs, Utilitas Math., 57, 121-128.

[12] C. J. Colbourn and J. H. Dinitz (2007). The CRC Handbook of Combinatorial Designs. 2nd ed., Chapman \& Hall, Boca Raton.

[13] L. Y. Deng and B. Tang (1999). Generalized resolution and minimum aberration criteria for Plackett-Burman and other nonregular factorial designs. Statistica Sinica, 9, 1071-1082.

[14] H. Ehlich (1964a). Determinantenabschätzungen für binäre Matrizen, Math. Z., 83, $123-132$.

[15] H. Ehlich (1964b). Determinantenabschätzungen für binäre Matrizen mit $N \equiv 3 \bmod$ 4, Math. Z., 84, 438-447.

[16] H. Ehlich and K. Zeller (1962). Binäre Matrizen. Z. Angew. Math. Mech., 42, T20$\mathrm{T} 21$.

[17] R. Fontana, G. Pistone and M. P. Rogantin (2000). Classification of two-level factorial fractions, Journal of Statistical Planning and Inference, 87, 149-172.

[18] Z. Galil and J. Kiefer (1980). D-optimum weighing designs. Annals of Statistics, 8, 1293-1306.

[19] J. Hadamard (1893). Résolution d'une question relative aux déterminants, Bull. Sci. Math., 2, 240-246.

[20] A. Hedayat and W. D. Wallis (1978). Hadamard matrices and their applications. Annals of Statistics, 6, 1184-1238.

[21] Q. M. Husain (1945). On the totality of the solutions for the symmetrical incomplete block designs: $\lambda=2, k=5$ or 6 . Sankhya, $7,204-208$.

[22] H. Kharaghani (1987). A construction of $D$-optimal designs for $N \equiv 2 \bmod 4$. J. Combin. Theory Ser. A 46, 156-158.

[23] S. Kounias, C. Koukouvinos, N. Nikolaou and A. Kakos (1994). The non-equivalent circulant $D$-optimal designs for $n \equiv 2 \bmod 4, n \leq 54, n=66$. J. Combin. Theory Ser. A, 65, 26-38.

[24] S. Kounias, C. Koukouvinos, N. Nikolaou and A. Kakos (1996). The non-equivalent circulant $D$-optimal designs for $n=90$. J. Statist. Plann. Inference, 53, 253-259.

[25] J. L. Loeppky, K. Bingham and R. R. Sitter (2006). Constructing non-regular robust parameter designs. Journal of Statistical Planning and Inference, 136, 3710-3729.

[26] R. Mukerjee and C. F. J. Wu (2006). A Modern Theory of Factorial Designs. Springer Series in Statistics. 
[27] A. M. Mood (1946). On Hotelling's weighing problem, Ann. Math. Statist., 17, 432446.

[28] C. Moyssiadis and S. Kounias (1982). The exact D-optimal first order saturated design with 17 observations. Journal of Statistical Planning and Inference, 7, 13-27.

[29] W. P. Orrick (2005). The maximal $\{-1,1\}$-determinant of order 15. Metrika, 62, $195-219$.

[30] W. P. Orrick (2008). On the enumeration of some D-optimal designs. Journal of Statistical Planning and Inference, 138, 286-293.

[31] M. O. Pavcevic and E. Spence (1999a). Some new symmetric designs with $\lambda=10$ having an automorphism of order 5. Discrete Math., 196, 257-266.

[32] M. O. Pavcevic and E. Spence (1999b). Some new symmetric designs, J. Combin. Designs, 7, 426-430.

[33] F. Pukelsheim (1993). Optimal Design of Experiments. Wiley, New York.

[34] D. Raghavarao (1959). Some optimum weighing designs, Ann. Math. Statist. 30, 295-303.

[35] J. Seberry and M. Yamada (1992). Hadamard matrices, sequences, and block designs, in Contemporary Design Theory, J. H. Dinitz and J. R. Stinson eds., pp. 431-560. Wiley, New York.

[36] W. D. Smith (1988). Studies in computational geometry motivated by mesh generation. Ph. D. dissertation, Princeton University.

[37] E. Spence (1993). Symmetric $(41,16,6)$-designs with a nontrivial automorphism of odd order. J. Combin. Designs. 1, 193-211.

[38] E. Spence (1995). Five nondual 2-(41,16,6) designs with a trivial automorphism group. Ars Combin. 41, 117-122.

[39] B. Tang and L. Y. Deng (1999). Minimum $G_{2}$-aberration for nonregular fractional factorial designs. Annals of Statistics, 27, 1914-1926.

[40] J. A. Todd (1933). A combinatorial problem. J. Math. Phys., 12, 321-333.

[41] K. W. Schmidt (1973). Problem 72-19, A bound for a $4^{k}$-order maximal $(0,1)$ determinant. SIAM Rev., 15, 673-674.

[42] J. J. Sylvester (1867). Thoughts on inverse orthogonal matrices, simultaneous signsuccessions, and tesselated pavements in two or more colours, with applications to Newton's rule, ornamental tile-work, and the theory of numbers. London Edinburgh and Dublin Philos. Mag. and J. Sci., 34, 461-475. 
[43] T. van Trung (1982). The existence of symmetric block designs with parameters $(41,16,6)$ and $(66,26,10)$, J. Combin. Theory Ser. A, 33, 201-204.

[44] J. Williamson (1946). Determinants whose elements are 0 and 1. Amer. Math. Monthly, 53, 427-434.

[45] W. Wojtas (1964). On Hadamard's inequality for the determinants of order nondivisible by 4, Colloq. Math., 12, 73-83.

[46] H. Xu (2003). Minimum moment aberration for nonregular designs and supersaturated designs. Statistical Sinica, 13, 691-708.

[47] H. Xu and C. F. J. Wu (2001). Generalized minimum aberration for asymmetrical fractional factorial designs. Annals of Statistics, 29, 1066-1077.

[48] C. H. Yang (1966a). Some designs for maximal $(+1,-1)$-determinant of order $n \equiv 2$ $(\bmod 4)$, Math. Comp., 20, 147-148.

[49] C. H. Yang (1966b). A construction for maximal $(+1,-1)$-matrix of order 54, Bull. Amer. Math. Soc., 72, 293.

[50] C. H. Yang (1968). On designs of maximal $(+1,-1)$-matrices of order $n \equiv 2(\bmod$ 4), Math. Comp., 22, 174-180.

[51] C. H. Yang (1969). On designs of maximal $(+1,-1)$-matrices of order $n \equiv 2(\bmod$ 4), II, Math. Comp., 23, 201-205.

[52] C. H. Yang (1976). Maximal binary matrices and sum of two squares, Math. Comp., 30, 148-153.

[53] K. Q. Ye (2003). Indicator function and its application in two-level factorial designs. Annals of Statistics, 31, 984-994. 